\title{
Criminologie
}

\section{Les grains de la colère. Géographie de l'émotion populaire en France au sujet de la cherté des céréales (1816-1817)}

\section{Denis Béliveau}

Volume 27, numéro 1, 1994

Analyse spatiale du crime

URI : https://id.erudit.org/iderudit/017350ar

DOI : https://doi.org/10.7202/017350ar

Aller au sommaire du numéro

Éditeur(s)

Les Presses de l'Université de Montréal

ISSN

0316-0041 (imprimé)

1492-1367 (numérique)

Découvrir la revue

Citer cet article

Béliveau, D. (1994). Les grains de la colère. Géographie de l'émotion populaire en France au sujet de la cherté des céréales (1816-1817). Criminologie, 27(1),

99-115. https://doi.org/10.7202/017350ar
Résumé de l'article

By examining protests over the high price of wheat, this article attemps to re-evaluate the true impact of the variable "price " on protest movements. Over and above the pressure exerted by the increase in the price of produce, other factors figured in increases in popular unrest. Oversimplification and a mechanistic analysis linking these protests with the high price of grain fail to explain the nature of these movements. It is necessary, therefore, to consider not only structural elements, but also the interplay of local unions, the impact of descriptions of self and other, and the influence of rumours and expectations. 


\title{
LES GRAINS DE LA COLÈRE. GÉOGRAPHIE DE «L'ÉMOTION POPULAIRE » EN FRANCE AU SUJET DE LA CHERTÉ DES CÉRÉALES (1816-1847). Denis Béliveau ${ }^{1}$
}

\begin{abstract}
By examining protests over the high price of wheat, this article attemps to re-evaluate the true impact of the variable "price protest movements. Over and above the pressure exerted by the increase in the price of produce, other factors figured in increases in popular unrest. Oversimplification and a mechanistic analysis linking these protests with the high price of grain fail to explain the nature of these movements. It is necessary, therefore, to consider not only structural elements, but also the interplay of local unions. the impact of descriptions of self and other, and the influence of rumours and expectations.
\end{abstract}

Mars 1816. «Une sorte d'insurrection populaire dont l'impunité nous ramènera infailliblement aux premiers désordres qui ont causé la révolution, vient d'avoir lieu en la commune de Tressin (...) Une fermière de Tressin, la Veuve Moutier de nom, fit un marché le 26 de ce mois avec le $S^{r}$ Augustin Harnou d'Orchies et convint de lui fournir, quand il viendrait avec son chariot et ses chevaux, vingt-quatre sacs de froment; Harnou s'est présenté le 27, a soldé l'importance de son marché, a exhibé son passavant pour Orchies et, commençant son chargement, fut injurié par plusieurs femmes réunies spontanément autour de sa voiture; les ayant haranguées à sa manière pour les rendre plus câlines, une d'elles se mit à frapper sur un chaudron, ce qui augmenta la réunion et dès lors, une grêle de pierres, de mottes de terre, de boue, succéda aux imprécations; forcé à la retraite Harnou rentre dans la ferme, réclame la présence de la fermière qui y accédant se présenta aux femmes rassemblées avec sa fille: le tumulte redouble, on injurie la $\mathrm{V}^{\mathrm{e}}$ Moutier, on l'accuse de participer à l'intention d'Hamou d'affamer le village, on se porte à des violences, on lui jette de la boue, on frappe à sang coulant, sa fille à la tête avec un sabot dont s'était armée une des insurgées - puis on monte sur la voiture, à l'envie, et l'on précipite les sacs de froment qui y avaient été chargés ${ }^{2}$.»

1. Stagiaire postdoctoral, Centre international de criminologie comparé, Université dc Montréal. Ce texte constitue une version remaniée d'un chapitre de ma thèse de doctorat, soutenue à Paris (École des Hautes Études en Sciences Saciales) en 1992. Je tiens a remercier les deux lecteurs sollicités par la revue pour leurs commentaires et suggestions, de mêne que Yves Otis pour son aide à la réalisation des cartes.

2. Archives Departementales du Nord, M 135.23. Lettre du juge de paix du canton de Lannoy, arrondissement de Lille, au préfet du Nord, 29 mars 1816. 
Ainsi commençait, pour les autorités chargées du maintien de l'ordre. une autre période d'intense activité occasionnée par la rareté et la cherté des grains. Certes, la relation présentée ci-haut exagère l'importance et le sens de l'événement; en effet, même si la tourmente révolutionnaire s'est accompagnée de nombreuses manifestations de ce type (Rudé, 1982), de tels mouvements de colère réapparaissent périodiquement depuis plus d'un siècle (L. Tilly, 1972; C. Tilly, 1986) et n'ont pas la portée épidémique qu'on leur prête ici. En ces temps où le pain quotidien signifie plus qu'une prière, les modalités du déclenchement de la violence demeurent somme toute assez classiques : une mauvaise récolte, un contexte de rareté, un geste mal interprété, des paroles imprudentes, et c'est «l'émotion populaire».

Comme au siècle précédent, de telles manifestations découlent généralement d'un même processus : à la suite de mauvaises récoltes, les grains sont rares et leur prix, à la hausse : certains individus (des marchands, des blatiers ou des cultivateurs) tentent alors d'en tirer profit d'une manière jugée abusive par les populations pauvres. C'est là du moins un sentiment partagé par les manifestants: les rumeurs de pénurie, de spéculation et d'accaparement ne tardent pas à se propager et à attiser les frustrations. Tel un orage, la colère populaire s'abat sur les détenteurs de grains.

Ces révoltes ont pour principales fonctions d'assurer à la communauté un approvisionnement en grains et ce, à un prix «juste». Pour cela, les manifestants font entrave à la circulation (ils interceptent les convois pour entreposer ou vendre les grains), «taxent» ceux-ci (on en fixe arbitrairement le prix) ou exercent des menaces et des pressions sur ceux qui détiennent du blé pour les amener à garnir les étaux du marché. En d'autres endroits, des perquisitions sont effectuées dans les greniers des cultivateurs et des marchands soupçonnés de se livrer à de la spéculation. Pillages, désordres au marché, ventes forcées et mendicité en réunion complètent le répertoire de la violence populaire.

Depuis près d'une vingtaine d'années, plusieurs travaux (Thompsoiin, 1971 ; Farge, 1974 et 1986 ; Rudé, 1982) sont venus renouveler l'appréciation portée envers ce type de soulèvements populaires, longtemps réduits à leur dimension instinctive et passéiste. Frustrés de ne pouvoir s'approvisionner en pain, les manifestants n'auraient écouté que leur ventre et leurs instincts. Tel était souvent le raisonnement guidant les premières analyses. Ces révoltes, il est vrai, renvoient à un mode de fonctionnement idéalisé où les autorités étaient censées pourvoir à l'approvisionnement en grains (Kaplan, 1988); par elles, on réactualise ces attentes. Rien d'étonnant dès lors, à ce que ces manifestations aient longtemps été perçues comme déphasées, et ce même par certains auteurs marxistes qui, tout en reconnaissant la sévérité des conditions de vie, ont privilégié une analyse de type socio- 
économique. Dès lors, la notion de «conscience politique» occupe une place centrale, et sur cette base, des distinctions sont établies: on parlera ainsi de révoltes préindustrielles pour qualifier ces formes de protestation (Hobsbawm, 1966).

À trop vouloir juger ces mouvements, à trop chercher à ne les aborder que sous l'angle de leur insertion dans l'évolution des modes de production (Bohstedt, 1988), certains historiens ont peut-être perdu de vue qu'ils impliquent une vision de l'ordre social, une autre façon de percevoir les rapports économiques (Thompson, 1971). Ces mouvements supposent également des rôles et des rapports de pouvoir qui se négocient d'abord à l'intérieur de la communauté et qui ne prennent sens que dans la logique des représentations collectives (Corbin, 1990 et 1991). Il importe en effet de tenter de saisir comment opèrent ces compréhensions, le rôle de la mémoire des résistances collectives, et leur éventuelle réinterprétation. La révolte n'est pas que réminiscence mais s'intègre bien à des identités sociales et à des bouleversements matériels et symboliques.

Ainsi, les différentes analyses portant sur ces mouvements ont longtemps établi un rapport très étroit entre la hausse des prix et le déclenchement de ces révoltes, comme si le conflit se réduisait à ce seul motif. À l'analyse cependant, cette équation (ou cette chaîne de causalité) appelle un certain nombre de nuances. En nous penchant sur la répartition géographique des révoltes recensées entre 1816 et 1847 (Béliveau. 1992), et en scrutant l'évolution du prix du blé dans les régions où ces désordres sont les plus fréquents, nous nous proposons ici d'examiner plus attentivement ce rapport, dans la perspective de mieux en cerner les modalités.

La distribution géographique de ces mouvements n'obéit pas à la seule pression qu'impose la cherté des grains. En effet, de telles manifestations ne se produisent pas nécessairement dans les régions où le prix du froment atteint les plus hauts taux: d'une crise à l'autre, on ne détecte pas de corrélation entre l'ampleur de la cherté du blé et l'intensité des révoltes. Les variations de prix, les habitudes de consommation liées aux structures agricoles régionales et au type de production privilégié, de même que l'existence de réseaux de communication permettant des échanges commerciaux sont autant d'éléments qu'il importe de considérer; en s'y référant, on s'explique mieux la localisation des troubles frumentaires. L'ensemble de ces données, combiné bien sûr à la hausse des prix, confèrent à la géographie des révoltes un éclairage plus juste que ce que suppose la seule variable "prix». En outre, les solidarités communales qui se développent autour de la question vivrière et les représentations que peut se faire la foule à l'égard des marchands de grains s'avèrent fondamentales pour comprendre ces mouvements. 
Finalement, l'effet d'entraînement. les craintes et les anticipations de certains consommateurs se révèlent être des facteurs tout aussi importants pour bien comprendre la concentration des révoltes autour de certains départements. Ces sentiments interviennent assurément dans la compréhension que les manifestants se font de la situation et des solutions à envisager.

\section{L'IMPACT RELATIF DE LA HAUSSE DU PRIX DES GRAINS}

En regroupant l'ensemble des manifestations rapportées entre 1816 et 1847 (voir carte 1), on constate une concentration des révoltes dans certains départements, situés principalement dans le nord-ouest et l'ouest de la France mais aussi dans le centre et le nord du pays. Les départements du nord-est et de l'est se révèlent être affectés dans une moindre mesure : seuls deux d'entre eux (l'Aube et la Saône-et-Loire) sont touchés par un nombre élevé de révoltes. À l'opposé, le sud demeure épargné par de tels mouvements. En effet. pratiquement tous les départements du bassin méditerranéen restent exempts de révoltes, alors que l'on n'en signale qu'un faible nombre dans le sud-ouest et le sud-est du pays.

Certes, on ne tient compte ici que des cas signalés aux autorités supérieures; nos données demeurent de ce fait incomplètes, en raison même des conditions de production de nos sources. En effet, malgré les recommandations émises, les divers représentants de l'appareil administratif ne signalent pas toujours ces désordres, les jugeant par trop insignifiants, ou croyant être en mesure de résoudre le conflit par leur seule médiation. En outre, les autorités locales (maires, juges de paix et gendarmes) partagent quelquefois les griefs de ceux qui s'en prennent aux marchands, d'où un certain atermoiement. Malgré ces lacunes, l'information disponible demeure assez contrastée pour être significative ${ }^{3}$.

Que comprendre de cette répartition des révoltes entre les différents départements? Examinons d'abord l'impact de la cherté des grains. Entre 1816 et 1847 , nous avons pu retracer 929 manifestations reliées à la question du blé. Les désordres se concentrent principalement autour de quatre périodes : 1816-1817, 1829-1830, 1839-1840 et 1846-1847. À chaque fois, il s'agit d'années caractérisées par de mauvaises moissons et par une hausse marquée des prix. Si la cherté du blé intervient assurément dans l'instauration d'un climat propice au déclenchement de telles révoltes, il est cependant nécessaire d'évaluer la portée réelle de ce facteur et de voir comment il agit à la lumière de la distribution géographique des manifestations.

3. En outre, l'absence de révolte dans les départements du Sud et de l'Est s'avère confirmée par d'autres sources. Les manifestations non signalées semblent avoir lieu dans les régions qui en comptent déjà beaucoup. On ne signale alors que les plus importantes, ou du moins celles jugées comme telles. 


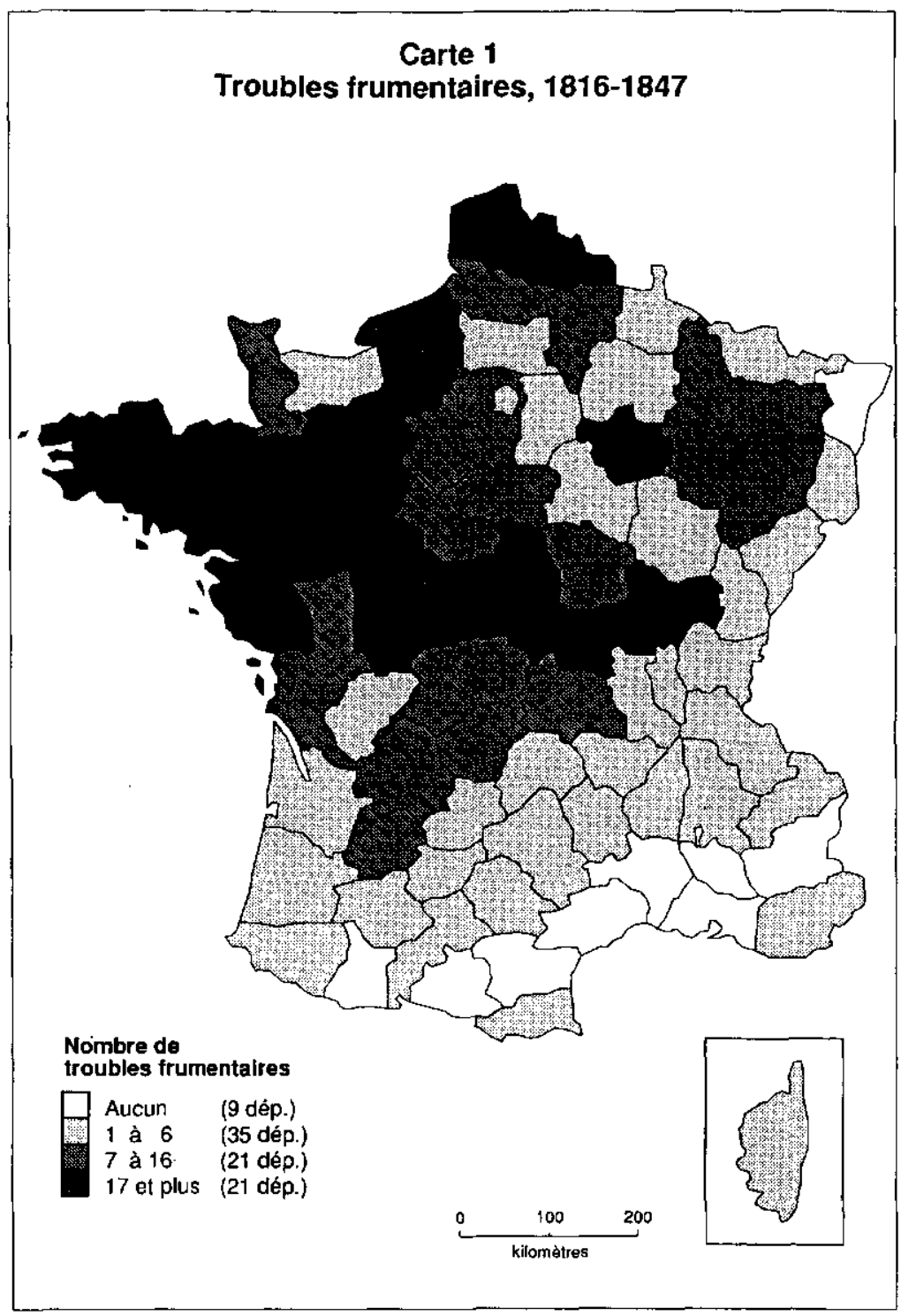


Précisons d'abord que les problèmes reliés aux mauvaises récoltes et à leurs conséquences économiques et sociales sont nombreux. surtout dans un pays dont l'économie repose encore principalement sur l'agriculture. Selon le schéma classique décrit par E. Labrousse (1933 et 1956), de mauvaises moissons provoquent une forte tension sur les prix, généralement aggravée par la spéculation, plongeant ainsi dans la misère de nombreux paysans (ceux qui produisent peu) et la plupart des salariés ruraux, nombreux à cette époque. En ces périodes de crises, les autorités tentent bien de venir en aide aux plus démunis par la création d'ateliers de charité ou en levant des souscriptions leur permettant d'offrir des bons de réduction pour le pain. Malgré ces mesures, de nombreuses familles, celles où l'on consacre une part importante du budget à l'achat de pain et de blé, s'avèrent être cruellement frappées.

Il importe en outre de bien comprendre que le marché des grains constitue encore au $\mathrm{XIX}^{\mathrm{e}}$ siècle un secteur d'activité caractérisé par une grande instabilité les variations de prix demeurent très importantes. Ainsi, entre le mois de janvier 1816 et la fin de l'année 1817 , le prix moyen de l'hectolitre de froment se chiffrait à 32,23 francs pour l'ensemble du pays alors qu'il ne s'élevait qu'à respectivement 17,73 fr. et 19,68 fr. lors des deux années précédentes (Labrousse, 1970). De fait, on enregistre des fluctuations mensuelles très prononcées. En janvier 1816, l'hectolitre de froment se négocie à 22,24 fr.; par la suite, il augmente de façon constante jusqu'en juin 1817 , où il atteint un sommet à 45,46 fr. (moyenne nationale).

Par-delà cette versatilité, des écarts considérables divisent le pays. À chacune de ces crises, les populations de certains dêpartements s'avèrent affectêes par des prix plus êlevés. Ainsi, toujours en 1816-1817, le taux moyen demandé pour un hectolitre de froment oscille entre un minimum de 23,78 fr. dans le département des Deux-Sèvres (Ouest) et un maximum de 41,05 fr. dans celui des Vosges (Nord-Est). De telles variations existent lors de chacune des périodes de hauts prix. En 1846-1847, le cours moyen de l'hectolitre de froment en France s'élève à 26,53 fr. Les taux extrêmes se rencontrent alors en Ille-et-Vilaine (Nord-Ouest) avec 22,23 fr. et sur les marchés du Haut-Rhin (Nord-Est) où il est de 30,52 fr. (Labrousse, 1970).

Tous ces écarts (annuels. mensuels, d'un département à l'autre) illustrent bien la fragilité de ce marché et les limites des échanges commerciaux : les prix se forment localement. Les réseaux de communication ne permettent pas encore des échanges rapides d'une région à l'autre (Price, 1972), susceptibles d'assurer un approvisionnement constant et partant, une plus grande stabilité dans les cours. On est loin d'une intégration parfaite à une économie de marché. Compte tenu des revenus des familles des classes populaires et de l'importance - réelle et symbolique - du blé, une telle élasticité des prix ne peut qu'avoir des conséquences tragiques. De fait, ce 
n'est qu'après de nombreuses hésitations et de multiples reculs que les autorités parviennent à imposer au $\mathrm{XIX}^{\mathrm{e}}$ siècle le libéralisme dans ce secteur de commerce (Binet, 1939; Kaplan, 1986).

Il faut bien voir toutefois qu'un prix du froment plus élevé ne se traduit pas nécessairement par un plus grand nombre de révoltes. Au contraire même. les départements les plus touchés par les révoltes sont rarement ceux où le cours du froment atteint les plus hauts taux.

Ainsi, à la suite de mauvaises moissons en 1816 , le prix du froment est à la hausse partout; d'importants écarts se remarquent cependant d'une région à l'autre. Les départements qui affichent les plus hauts taux se concentrent près des frontières à l'est du pays et dans toute la région du Sud-Est. Le froment demeure moins cher en Bretagne, dans l'Ouest et le Centre. En fait, malgré le contexte particulier que suscitent les mauvaises récoltes, ces disparités régionales semblent respecter les différences habituellement enregistrées au niveau des prix du froment (Demonet, 1985). Or, comme l'illustrent les cartes 2 et 3 , la répartition départementale des troubles frumentaires de 1816-1817 ne recouvre pas les régions de cherté absolue. Il n'y a qu'un seul département (Saône-et-Loire) fortement touché à la fois par le nombre de révoltes et par de plus hauts prix. Non seulement relève-t-on peu de manifestations dans les régions où sévit le plus durement la cherté des grains mais il y a presque corrélation inverse. Les taux atteints par le froment s'avèrent en effet moins élevés dans les départements où un grand nombre de mouvements de protestation sont rapportés : Ille-etVilaine, Mayenne, Maine-et-Loire, Indre-et-Loire, Loiret, Cher, etc. En 1846-1847, les régions les plus touchées par la cherté du blé varient quelque peu, les populations du Centre et du bassin parisien étant alors affectées par des prix plus élevés qu'en 1816-1817. Mais encore là, les contrées où les mercuriales font état des plus hauts taux ne recoupent que très rarement ${ }^{4}$ celles qui sont les plus perturbées par des révoltes (voir cartes 4 et 5 ) $^{5}$.

4. On ne relève que quelques exceptions où se vérifie cette relation : c'est le cas pour le Saône-et-Loire en 1816-1817 et en 1846-1847, pour le Nord en 1829-1830 et pour l'Indre en 1846-1847.

5. Pour la réalisation des cartes concernant le prix du froment en 1816-1817 et 1846-1847 (les cartes 3 et 5), nous avons établi, sur la base des mercuriales (Labrousse, 1970) le taux moyen annuel d'un hectolitre de froment pour chaque département. Suivant les résultats obtenus, nous avons procédé à une distribution autour d'une médiane, ce qui nous permet d'établir une građation de la cherté. Quatre paliers ont été déterminés suivant la cherté relative du froment. Une même démarche a été suivie pour l'exécution des cartes concernant la localisation des troubles à chaque période. 


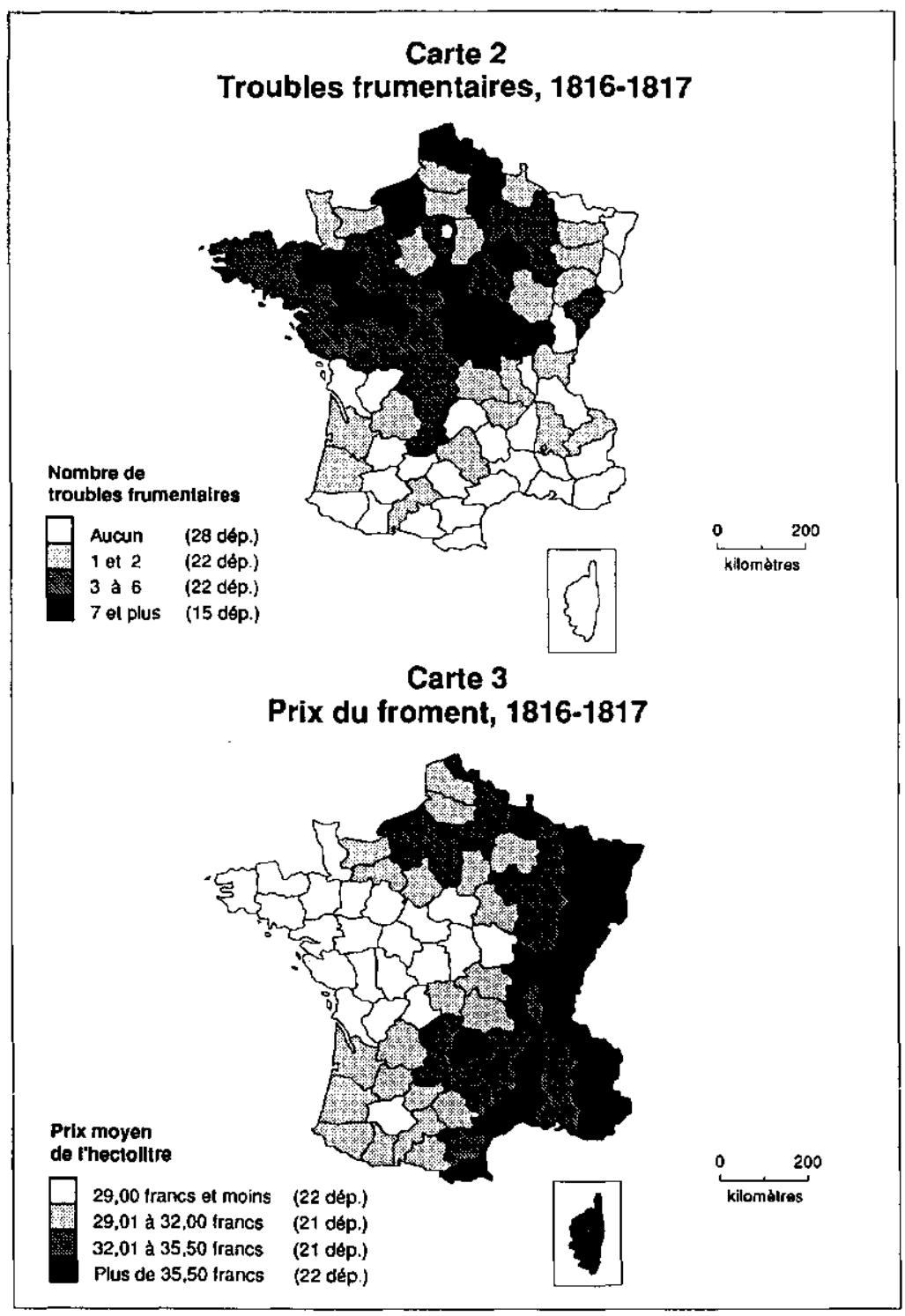




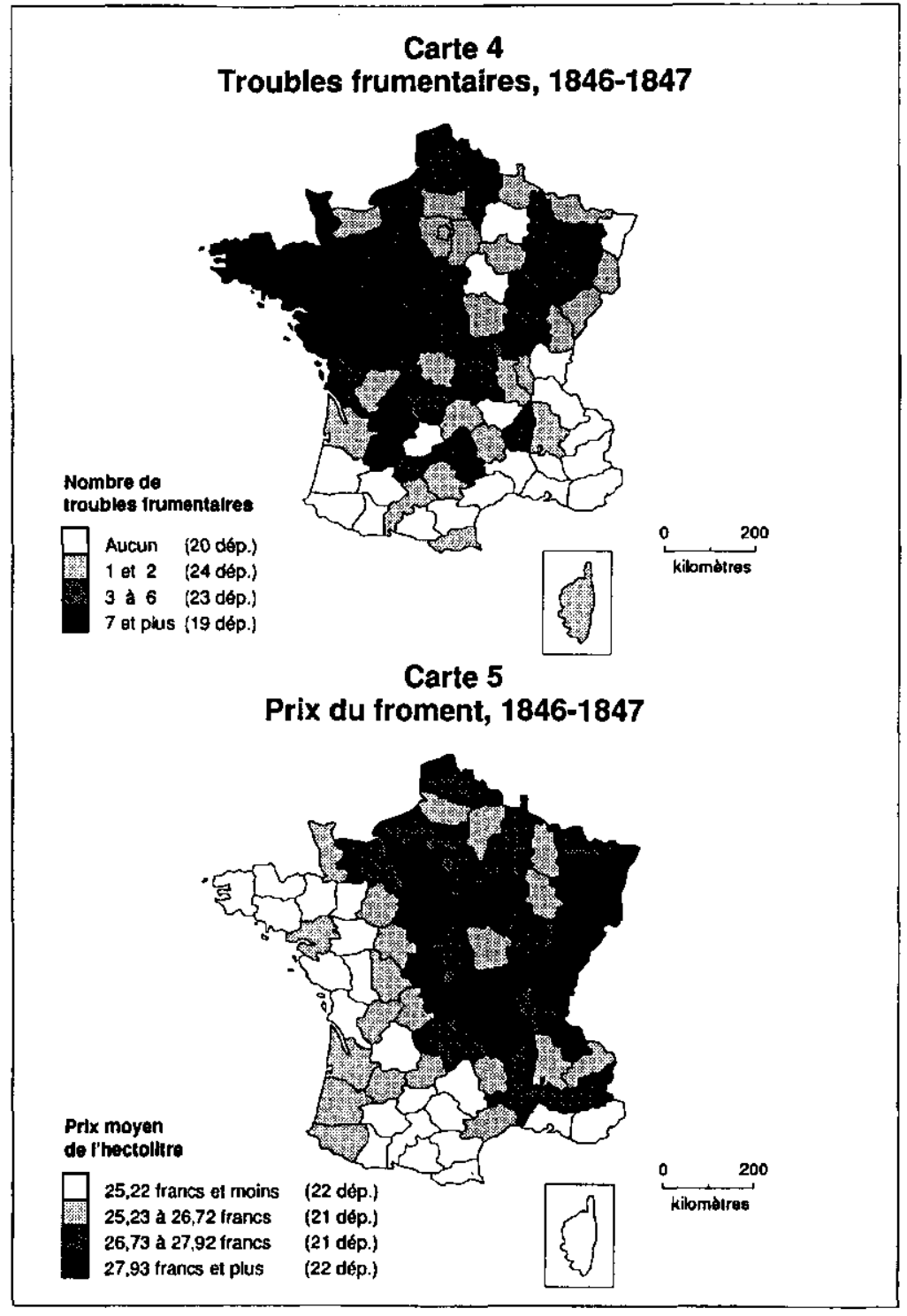




\section{D'AUTRES VARIABLES}

Certes, on ne parle ici que du prix du froment alors que les populations pauvres, les plus susceptibles finalement de participer aux révoltes, n'en consomment que très rarement en raison de son coût plus élevé. Néanmoins, nos données peuvent être considérées comme représentatives de la situation générale qui prévaut au chapitre de la cherté des grains; d'une part le cours des autres grains (méteil, seigle et sarrasin) oscille suivant un même canevas; les zones de cherté de ces céréales sont souvent les mêmes que pour le froment, à quelques nuances près (Demonet, 1985, p. 405). D'autre part, en ces périodes. nombreux sont les acheteurs devant se rabattre (en raison de la pénurie et des prix) sur les céréales moins «nobles», accentuant ainsi la demande pour les autres céréales ce qui, du coup, contribue à leur rareté et favorise de nouvelles hausses. En outre, cette mutation vers d'autres denrées n'est pas toujours possible; en 1846, les récoltes de pommes de terre s'avèrent désastreuses un peu partout en France et celles du sarrasin le sont également dans certaines régions. Ces denrées constituent souvent le dernier recours des ménages les plus démunis.

Les crises cycliques de l'économie agricole ont donc des effets multiples. Certes. la misère, source de troubles, est gennérale. Pourtant, les mouvements de révoltes se concentrent plus particulièrement dans certaines régions qui ne se révèlent pas être celles où les prix sont les plus élevés. D'autres facteurs doivent donc intervenir. Si le cours des grains demeure à l'origine de la plupart des révoltes, leur concentration dans quelques régions, et à certaines périodes de l'année, s'expliquerait peut-être mieux par les inquiétudes que font naître de brusques augmentations. Plus que dans la cherté absolue des grains, les motifs pouvant expliquer ces manifestations résideraient plutôt du côté des variations de prix d'un mois à l'autre et des soubresauts d'un marché instable. Encore là, tout n'est pas si simple.

Ainsi en examinant pour chacune des périodes les mois "privilégiés" des révoltes, on se rend bien compte qu'ils ne coïncident pas nécessairement aux sommets atteints par les prix lors de chaque crise (Béliveau, 1992, p. 84). Généralement, c'est en mai et juin que la flambee des prix atteint son paroxysme; pourtant ces mois ne sont pas toujours les plus marqués par la grogne populaire. En 1847, c'est en janvier qu'éclate le plus grand nombre de révoltes; la période comprise entre mai et août s'avère somme toute assez calme. Il est vrai cependant que la répression, parfois très sévère, des troubles du début de l'année a pu exercer une certaine emprise. Au total, l'influence exercée sur le déclenchement des révoltes par la période de l'année et ses contraintes doit être relativisée. Certes, les mois de soudure entre deux récoltes demeurent un moment particulièrement critique en ce qui concerne l'approvisionnement; surtout lors des années de mauvaises moissons. Dès la fin des récoltes cependant, la population peut 
pressentir si l'année sera difficile et comment évoluera le prix des grains; il suffit alors parfois d'une rumeur, d'une remarque mal placée, d'une étincelle, pour qu'apparaissent des désordres qui, très souvent, se multiplieront.

\section{LES «PAYS » DE LA RÉVOLTE}

À première vue, la répartition de l'ensemble des révoltes sur le territoire peut paraître surprenante. D'une crise à l'autre, les régions les plus touchées ne s'avèrent pas être celles où le prix des grains atteint les plus hauts taux. En outre, cette concentration des manifestations dans le nord, le centre, l'ouest et le nord-ouest du pays ne constitue pas une illustration ou la marque d'une opposition entre une France "riche» ou mieux développée et une autre, plus pauvre. Comment expliquer en effet que le Nord et le Pas-de-Calais, l'Aube et certains des départements normands, généralement considérés cornme plus modernes (relativement bien industrialisés, dotés d'une agriculture compétitive) se retrouvent dans le même groupe que la Bretagne, si archaïque suivant les mêmes critères d'évaluation. Depuis les travaux de A. D'Angeville au XIX ${ }^{\mathrm{e}}$ siècle (rééd. 1964), nombreux ont été les auteurs à proposer l'existence de fortes distinctions entre la France du Nord et celle du Sud, y trouvant même parfois l'explication à certains comportements (mceurs, criminalité, etc. $)^{6}$. Mais cette opposition de part et d'autre d'une médiane qui relierait St-Malo à Genève semble peu opérationnelle en ce qui nous concerne ici.

En fait, pour établir un découpage de la France en fonction de la distribution des troubles frumentaires du XIXe siècle, il conviendrait plutôt de tracer une frontière le long d'un axe reliant La Rochelle à Genève. Les départements les plus touchés par ces révoltes se situent pour la plupart au nord de cette ligne alors que ceux au sud restent, en règle générale, peu affectés. Cette France contrastée à l'égard du conflit frumentaire pose cependant un problème. Une telle distribution a pour résultat de regrouper des espaces économiques fort différents; se côtoient, dans chaque cas, des zones de pauvreté et des régions dont le développernent est plus avancé. En fait, les variables habituellement utilisées pour mesurer les progrès agricoles et économiques demeurent peu efficaces pour analyser la géographie des troubles frumentaires. Il n'y a pas de corrélation entre les régions les moins bien nanties et un plus grand nombre de révoltes. Ainsi, la Bretagne et les zones montagneuses du Sud, l'essentiel des Pyrénées et les deux départements des Alpes s'intègrent aux zones de pauvreté agricole (Demonet.

6. Dès le $\mathrm{XIX}$ siècle, l'analyse des statistiques criminelles donne ainsi lieu à différentes tentatives pour démontrer la corrêlation qui existe entre les caractéristiques de la population laborieuse et les indices de criminalité. Voir G. Leclerc (1979), M. Morineau (1968). 
1985 p. 463 et ss.). Pourtant. si la première est l'une des régions les plus affectées par les révoltes, la tranquillité règne dans les autres. Inversement, même si l'agriculture pratiquée dans le nord de la France et celle qui s'est développée dans le bassin méditerranéen présentent des traits forts différents, les résultats en font néanmoins des contrées «modernes», privilégiées à certains égards; or ces deux régions s'opposent quant à l'importance des révoltes frumentaires: beaucoup dans la première, très peu dans l'autre.

\section{OPPOSITION VILLE-CAMPAGNE}

Que comprendre alors de cette répartition? Notons tout d'abord la singularité de Paris : le département de la Seine demeure peu affecté alors que tout autour se dressent des zones progressivement agitées à mesure que l'on s'éloigne de la capitale. Le cas de Paris n'est pas fortuit; les grandes villes (Paris, Marseille, Lyon, Lille) se révèlent peu touchées par ces mouvements ${ }^{7}$. Il faut toutefois souligner que les autorités de ces villes sont peut-être plus attentives aux problèmes des subsistances; ces dernières comptent en outre sur une forte présence policière (Price, 1982). Il est vrai également que conscientes du danger, certaines administrations municipales prennent des mesures pour soulager la misère des plus pauvres (en stimulant l'activité des négociants locaux, en créant des ateliers de charité ou en organisant des collectes de fonds) ${ }^{8}$. Par ailleurs, cette tranquillité ne surprend guère dans la mesure où les populations urbaines demeurent finalement tributaires des campagnes environnantes pour leur approvisionnement en grains. L'un des objectifs de ces révoltes - mettre obstacle à la circulation des grains - ne peut prendre de sens dans un contexte urbain.

Dans les autres grandes villes du pays, cette forme de violence reste assez rare et prend une couleur, des contours quelque peu différents. Rennes, Bordeaux, Reims. Toulouse et Nantes sont à peine affectees par quelques manifestations qui prennent, le plus souvent, la forme de désordres au marché ou de placards ${ }^{9}$. Contrairement à ces grands centres, les

7. Certes, la ville de Lille fait partie d'un département fort agité ; les troubles, cependant, se produisent en très grande majorité assez loin de la capitale du Nord.

8. En outre, certains grands patrons (comme Schneider au Creusot par exemple) sont intervenus directement pour assurer a leurs ouvriers un approvisionnement suffisant, et se préserver ainsi de possibles manifestations de mécontentement (Léveque, 1983).

9. Deux exceptions sont cependant notables. À Rennes, les 9 et 10 janvier 1847 , eclate une révolte d'une assez grande ampleur. Deux bateaux chargés de grains sont pilles et quelques boulangeries sont la proie de la violence populaire. Toulouse connaît également un soulèvement assez violent en novembre 1816. Le commerce y est alors perturbé pendant plus de trois jours. 
gros bourgs ou petites villes deviennent souvent le théâtre de révoltes : au total, une sur quatre se produit dans un chef-lieu d'arrondissement. C'est que dans ces agglomérations, les occasions sont multiples: souvent passages obligés pour les convois de grains, ces petites villes se transforment fréquemment en un lieu central pour l'échange et le commerce des grains. D'importantes halles s'y tiennent, de nombreuses transactions s'y effectuent, ce qui attire marchands. blatiers et populations des communes voisines. Lieux de négoce ou points de passage pour les convois, les conditions s'avèrent réunies pour le déclenchement de désordres. Le plus souvent cependant, c'est dans les petites communes, ou entre ces bourgades (sur les routes, chemins ou voies navigables), qu'éclatent les troubles frumentaires.

Force est donc de constater que le terrain de ces révoltes est principalement rural. C'est en vain cependant qu'on essaierait de faire des corrélations entre les zones de troubles frumentaires et les caractéristiques habituelles concernant les structures agricoles: faire-valoir, mode de tenure, superficie des exploitations, nombre de journaliers, de domestiques. etc: en outre, il n'y a pas d'adéquation entre les régions les plus industrialisées et l'ampleur de ces mouvements.

En fait la logique à la base de la répartition des révoltes sur le territoire semble s'articuler autour de trois éléments : production. consommation et distribution des grains. Dans les régions où les révoltes sont si nombreuses, la production agricole est avant tout frumentaire (Cobb, 1970: Béliveau, 1992). Ces régions ont ceci en commun qu'on y produit du blé - que ce soit du froment, du seigle, du méteil, du sarrasin - en quantité plus ou moins importante selon le développement des techniques agricoles (Demonet, 1985). Il semble que là où la production céréalière suffit habituellement à pourvoir aux besoins de la population locale, les réactions s'avèrent plus violentes face à la pénurie des grains; la suspicion et le mécontentement $s^{\prime} y$ développent rapidement. Comme s'il s'agissait d'une sorte de droit acquis, on accepte mal de voir partir les grains ou de subir une hausse importante de leur prix. Les zones les plus agitées semblent en effet être celles dont la production agricole, centrée sur les céréales, arrive tout juste à suffire aux besoins locaux. Il s'agit là d'une caractéristique importante. En fait, tout se passe comme si l'habitude d'avoir des grains en quantité suffisante se traduisait par une sensibilité différente pouvant se transformer plus facilement en révolte. Dans plusieurs de ces régions, les craintes de disette semblent plus vives, ce qui aurait pour effet de susciter un sentiment de panique lors des mauvaises récoltes. D'ailleurs, la part prépondérante des céréales dans les cultures (l'impérialisme frumentaire) découle souvent de telles appréhensions et constitue dans certains cas un frein aux changements. 
Dans ces contrées, la disette ou la seule crainte de manquer de grains actualise des comportements traditionnels qui reposent sur une représentation idéalisée de la marche du commerce : l'Etat doit assurer un approvisionnement constant et à un prix raisonnable tandis que les producteurs et marchands sont tenus d'offrir au marché les grains produits localement.

À ce chapitre, la particularité des départements affectés par un grand nombre de révoltes et situés plus au nord (en Normandie, autour du Pas-deCalais et du Nord) réside peut-être dans l'importance des populations qui y vivent; les besoins de celles-ci sont tels que même les hauts rendements de la production locale n'arrivent que difficilement à les combler. À cette pression démographique qui agit sur la demande en grains, $s^{*}$ ajoute la présence de voies de communication (terrestres ou fluviales) qui, en multipliant les passages de convois, suscitent une exacerbation des populations qui en manquent. On comprend mieux ainsi la cartographie des révoltes autour de Paris, dont les besoins occasionnent une intense activité des blatiers (Kaplan, 1988).

Les craintes, rumeurs et défiances de tout genre semblent donc se développer plus facilement dans ces régions où l'économie agricole est centrée sur les grains, et où ils circulent en grande quantité. Les régions de hauts prix céréaliers sont celles où il y a déficit constant de la production céréalière locale. La pénurie relative des grains (année normale) se traduit ici par des habitudes alimentaires différentes et une sensibilité autre, face à la question frumentaire. Ces régions ont recours à des approvisionnements en blé extérieurs, d'où peut-être une plus grande tolérance à l'égard des variations de prix. En outre. les revenus y sont généralement supérieurs et permettent une alimentation plus diversifiee, rendue possible par une production locale moins centrée sur les grains (Demonet, p. 415).

Certes, il ne s'agit que d'un modèle demandant un certain nombre de nuances. On aurait tort de centrer l'analyse sur un jeu de corrélations incapable de rendre compte de relations sociales et des conflits propres aux différentes communautés où éclatent ces désordres. Il est manifeste toutefois que la hausse des prix en période de mauvaises récoltes n'éveille pas les mêmes réactions d'une région à l'autre. Dès lors, si la cherté des grains reste un élément important dans le déclenchement de ces révoltes, il faut bien voir que le lien qui existe entre la cherté des grains et les révoltes demeure diffus et, somme toute, indirect.

\section{DIFFUSION DES PEURS}

Un dernier aspect concernant la géographie des révoltes mérite d'être souligné. Si les départements les plus affectés par les révoltes se situent généralement au nord d'un axe reliant La Rochelle à Genève, ce ne sont pas 
nécessairement toujours les mêmes d'une période à l'autre. On remarque ainsi que certains de ceux identifiés parmi les plus perturbés, en cartographiant l'ensemble des révoltes (voir carte 1), ne sont nullement affectés lors de certaines crises. Une analyse plus fine de la localisation des révoltes pour chacune des périodes s'avère révélatrice d'un autre facteur non négligeable, à savoir le «relais des peurs et des paniques» (Corbin, 1991).

En suivant le déclenchement chronologique des révoltes (Béliveau, 1992, p. 104 et ss.) on remarque en effet que d'une crise à l'autre, de nombreuses zones contiguës sont perturbées avec la même ampleur. Les départements affectés par un plus grand nombre de révoltes sont rarement isolés les uns des autres; ils forment entre eux des îlots dont la configuration change néanmoins suivant les périodes. En aucun cas sommes-nous en présence d'un département fort agité en côtoyant d'autres non perturbés par des manifestations. Une sorte de gradation existe entre eux.

L'effet d'entraînement se manifeste de façon locale et régionale. À maintes reprises, les révoltes se concentrent sur de courtes périodes (parfois deux à trois semaines) et dans un même arrondissement. Par les bruits que suscitent ces mouvements, les tensions débordent fréquemment sur les départements voisins, où l'on fait état d'une nouvelle série de troubles. Ces différentes vagues de manifestations attisent les sentiments de peur ${ }^{10}$, d'où les éclats successifs régionaux mais aussi un déséquilibre au niveau des approvisionnements, de nouvelles craintes, etc. D'une certaine façon, le tout s'enchaîne.

L'ampleur des troubles dans quelques départements découle donc parfois d'un effet «boule de neige» que les répercussions d'une seule manifestation peuvent susciter. Cette impulsion se nourrit des rumeurs, si importantes dans le déclenchement de ces désordres; une solidarité se développe alors contre les marchands, propriétaires ou négociants. De réels besoins, l'enthousiasme créé par l'événement et la conviction d'agir en toute légitimité complètent les sentiments des manifestants. Plus qu'un simple phénomène de mimétisme, ou de contagion, ces relais doivent être compris comme l'expression d'attentes et de façons de percevoir. Persuadé que les augmentations sont dues à des manœuvres illicites, on s'interpose comme on soubaiterait voir les autorités le faire. De fait, on décèle dans la plupart de ces révoltes, des gestes, des demandes et des rites qui tendent à montrer que la foule réagit en fonction de représentations et de rôles bien définis qui s'articulent autour de ce que E. P. Thompson (1971) désignait par «économie morale de la foule».

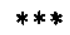

10. À ce chapitre, on assiste à un phénomène semblable à celui étudié par G. Lefebvre dès 1932 (rếd. 1970). 
En croisant la géographie des troubles et leur chronologie, on perçoit bien l'importance de l'impulsion donnée par certaines manifestations. Les relais qu'empruntent ces émotions populaires varient au gré des crises : c'est ce qui explique pourquoi les départements les plus perturbés ne sont pas toujours les mêmes d'une période à l'autre. En fait, dans ce type de désordres, l'important est souvent moins la réalité de la disette que l'idée que l'on s'en fait ; cette lecture, qui participe d'un entrelacs de rumeurs et d'attentes bien précises, semble souvent conditionner les comportements. Si de la peur du manque, naît le réflexe de faire des provisions, la révolte n'en est ici qu'une autre expression; à la différence près que l'on s'en prend aux personnes jugées responsables de cette situation de pénurie.

La géographie des révoltes demeure révélatrice de la force et de la logique de ces représentations. Celles-ci ont un «terrain» de prédilection: les départements où la production céréalière répond habituellement aux besoins des populations qui y vivent et où le blé s'impose comme une composante très importante de l'alimentation. Dans ces régions, la hausse des prix, les mécanismes qui suscitent ces augmentations et la compréhension que s'en font certains consommateurs, demeurent plus déterminants que les sommets atteints par les mercuriales. Leurs craintes se nourrissent des premières hausses, de l'effet d'anticipation et des rumeurs; le seul passage d'un convoi de grains, d'un étranger, ou d'un marchand suffit souvent à jeter l'émoi. De là, tout est possible. La cherté est donc la toile de fond; elle n'opère que de façon diffuse. En fait, les motifs auxquels se rattachent ces troubles frumentaires s'ancrent autour d'une réalité complexe que la simple équation cherté $=$ révolte arrive très mal à traduire.

\section{BIBLIOGRAPHIE}

BÉLIVEAU, D. (1992), Les révoltes frumentaires en France dans la premiere moitié du XIX siècle. Une analyse des rapports de sociabilité, de la distribution des rôles sexuels et de leurs impacts sur la répression des désordres, Paris, thèse de doctorat (nouveau régime), École des Hautes Études en Sciences Sociales.

BINET, P. (1939), La réglementation du marché du blé en France au XVIII siècle et à l'époque contemporaine, Paris, Librairie sociale et économique.

BOHSTEDT, J. (1988), «Gender, Household and Community Politics: Women in English Riots, 1790-1810», Past and Present, 120, p. 88-122.

COOB, R. (1970), The Police and the People. French Popular Protest (1789-1820), N.Y., Oxford University Press.

CORBIN, A. (1990), Le village des cannibales, Paris, Aubier.

CORBIN, A. (1991), «L'histoire de la violence dans les campagnes françaises au $\mathrm{XIX}^{e}$ siècle », Ethnologie française, XXI, p. 224-236. 
D'ANGEVILLE, A. (1969), Essai sur la statistique de la population française, réimpression avec préface de E. Le Roy Ladurie, Paris, Mouton.

DEMONET, M. (1985), Tableau de l'agriculture française au milieu du XIXe siècle. $L^{\prime}$ 'enquête de 1852, Paris, thèse de doctorat de $3^{\mathbf{2}}$ cycle, École des Hautes Éludes en Sciences Sociales.

FARGE, A. (1974), Le vol d'aliments a Paris au XVIIF siecle, Paris, Plon.

FARGE, A. (1986), La vie fragile. Violence, pouvoirs et solidarités d̀ Paris an XVIII siecle, Paris, Hachette.

HOBSBAWM, E.J. (1966), Les primitifs de la révolte dans l'Europe moderne, trad. de R. Laars, Paris, Fayard.

KAPLAN, S. (1986), Le Pain, le Peuple et le roi : la bataille du libéralisme sous Louis $X V$, Paris, Librairie académique Perrin.

KAPLAN, S. (1988), Les ventres de Paris. Pouvoir et approvisionnement dans la France d'Ancien Régime, trad. de S. Boulongne, Paris, Fayard.

LABROUSSE, E. (1933), Esquisse du mouvement des prix er des revenus en France au XVIIT siècle, Paris, Presses Universitaires de France.

LABROUSSE, E. (dir.) (1956), Aspects de la crise et de la dépression de l'économie française au milieu du XIX ${ }^{e}$ siècle, Paris, Bibliothèque de la Révolution de 1848.

LABROUSSE, E. (1970), Le prix du froment en France (1726-1913), Paris, S.E.V.P.E.N.

LEFEBVRE, G. (1970), La Grande Peur de 1789, rééd. avec préface de J. Revel, Paris, Armand Colin.

LÉVÊQUE, P. (1983), Une sociêté en crise : la Bourgogne au milieu du XIX $X^{e}$ siècle (1846-1852), Paris, éd. de l'É.H.É.S.S.

MORINEAU, M. (1968), «Y a-t-il eu une révolution agricole en France au XVIII siècle? ", Annales E.S.C., 27, p. 299-326.

PRICE, R. (1972), The French Second Republic. A Social History, Ithaca, Cornell University Press.

PRICE, R. (1982), «Techniques of Repression : the Control of Popular Protest in Mid-Nineteenth-Century France », Historical Journal, 25, p. 859-887.

RUDE, G. (1982), La foule dans la Révolution française, trad. de A. Jordan, Paris, Maspero.

THOMPSON, E.P. (1971), «The Moral Economy of the English Crowd in the Eighteenth Century", Past and Present, 50, p. 76-136.

TILLY, C. (1986), La France conteste, trad. de E. Diacon, Paris, Fayard.

TILLY, L. (1972), «La révolte frumentaire, forme de conflit politique en France», Annales E.S.C., 27, p. 731-757. 